including any laboratory investigations. Hence this study aimed to identify high risk clincial parameters helpful in Predicting outcome of hospitalised neonates.

Design and setting Prospective, Clinical, teaching hopital based observational study.

Methods All the 344 neonates at admission were assessed on the basis of various clinical parameters.

Statistical analysis

Comparision between means by ANOVA. Odds ratio with 95\% CI. Multiple logistic regression analysis, Positive and negative predictive values, sensitivity, specificity of each significant variable. significance level $\mathrm{p} \leq 0.05$.

Results Variables significantly associated with mortality were: HR (OR-3.27, CI -1.56-6.83, p-0.0016), RR (OR-5.61, CI 2.26-13.96, p-0.0002), SPO2 (OR-12.17, CI -4.60-32.72, p$<0.0001$ ), CFT (OR-24.31, CI -7.39-79.94, p- < 0.0001), hypo/ hyperthermia (OR-3.58, CI -1.66-7.70, p-0.001), birth weight (OR-2.13, CI -1.05-4.33, p-0.037), sensorium (OR-21.07, CI$8.30-53.48, \mathrm{p}-0.0001$ ), activity (OR-44.55, CI -6.01-330.26, p0.0002 ), pallor (OR-0.15, CI $-0.07-0.33$, p- $<0.0001$ ), cyanosis (OR-0.10, CI -0.04-0.25, p-0.0001), bleeding (OR-0.29, CI 0.10-0.80, p-0.016), dehydration (OR-4.70, CI-1.99-11.09, p$0.0004)$, respiratory distress (OR-2.43, CI -1.18-4.96, p-0.015), murmur (OR-0.25, CI -0.09-0.70, p-0.008), abdominal distension (OR-0.16, CI $-0.07-0.33$, p- $<0.0001$ ), hepatomegaly (OR0.07 , CI -0.02-0.17, p-0.0001), tone (OR-37.12, CI -8.66158.99, p-0.0001)/ (OR-18.2, CI-2.32-142.91, p-0.005), absent Moro's (OR-14.43, CI-5.74-36.28, p-0.0001).

Conclusions Outcome of neonates can be predicted at the time of admission, using simple, easily assessed bedside clinical parameters.

\section{PO-0708 IDENTIFICATION OF HIGH RISK CLINICAL PARAMETERS FOR PREDICTING OUTCOME OF HOSPITALISED NEONATES-A PROSPECTIVE OBSERVATIONAL STUDY}

${ }^{1} \mathrm{H}$ Singh, ${ }^{2}$ RK Soni, ${ }^{1} \mathrm{G}$ Singal. 'Pediatrics, Dayanand Medical College and Hospital, Ludhiana, India; '2Community Medicine, Dayanand Medical College and Hospital, Ludhiana, India

\subsection{6/archdischild-2014-307384.1344}

Background and aims The early identification of severity of illness is important for prioritising treatment to reduce mortality and morbidity in neonates but it is sometimes difficult to assess. An overall subjective assessment of the severity of underlying illness is useful in final decision making. Most of the available neonatal scoring systems have certain limitations namely : i. These are not a one time asessment and data collection extends over a number of hours. ii. Are complex, labour intensive, expensive and include a large number of investigations. iii. Limited to certain category of neonates.

None of the scoring systems can predict neonatal outcome by assessing only clinical parameters without including any laboratory investigations. Hence there is a need for a study to predict neonatal outcome at admission. involving only clinical parameters. This study aimed to identify high risk clincial parameters that will be helpful in Predicting outcome of hospitalised neonates.

Material

Design and Setting Prospective, Clinical, teaching hopital based observational study.

Subjects A total of 344 neonates included, at admission.

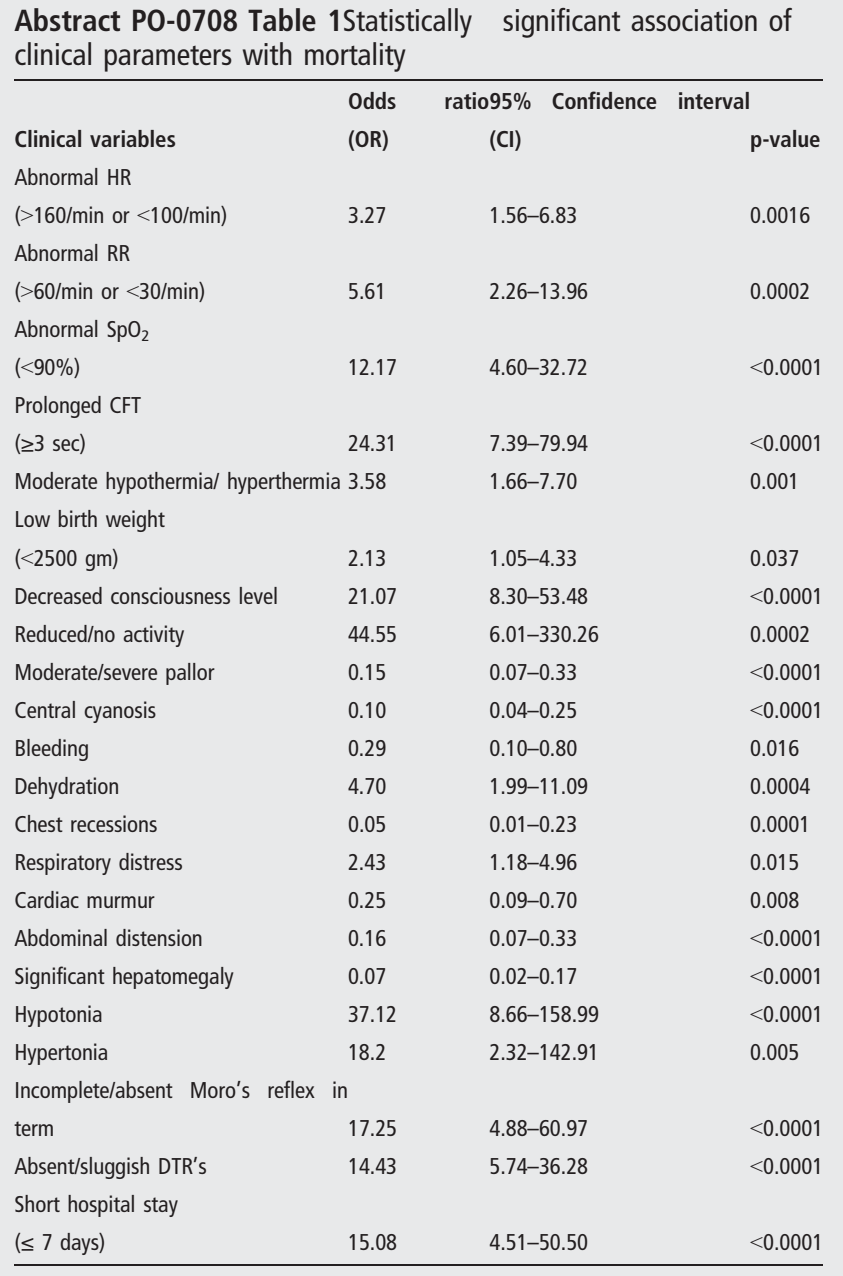

Methods All the neonates at admission were assessed on the basis of various clinical parameters and history. These included vital sign (HR, RR, CFT, Temp), activity, cry, pallor, icterus, cyanosis, bleeding, anterior fontanel, congenital malformations, respiratory distress, cardiac murmur, hepatomegaly, neonatal reflexes, weight, gestational age, seizures.

Statistical analysis Mean and SD were computed; comparision between mean was done by ANOVA. Odds ratio with 95\% CI were calculated for each parameter. Multiple logistic regression analysis was carried out Positive and negative predictive values, sensitivity, specificity of each significant clinical variable were calculated. The significance level was taken as $p$ value $\leq 0.05$.

Results Variables which were significantly associated with outcome in terms of mortality on univariate analysis (Table) were: $\mathrm{HR}, \mathrm{RR}, \mathrm{SPO}_{2}$, CFT, hypo/hyperthermia, low birth weight, abnormal conciousness level, reduced or no activity, moderate or severe pallor, cyanosis, bleeding, dehydration, respiratory distress, cardiac murmur, abdominal distension, hepatomegaly, hypo/hypertonia incomplee/absent Moro's/DTRs and hospital stay of $<7$ days.

On multiple logistic regression the significant independent clinical parameters for mortality were : $\mathrm{HR}, \mathrm{RR}, \mathrm{O}_{2}$ saturation, CFT, hypo/hypothermia, LBW, decreased sensorium level, reduced or no activity, moderate/severe pallor, cynosis, bleeding, dehydration, respiratory distress, cardiac murmur, abdominal distension.

Conclusions Outcome of neonates can be predicted at the time of admission, using simple, easily assessed bedside clinical parameters. 